\title{
MEASURING THE ABUNDANCE OF SUB-KILOMETER-SIZED KUIPER BELT OBJECTS USING STELLAR OCCULTATIONS
}

\author{
Hilke E. Schlichting ${ }^{1,2,8}$, Eran O. Ofek ${ }^{3}$, Re’em Sari $^{4}$, Edmund P. Nelan ${ }^{5}$, Avishay Gal-Yam $^{3}$, \\ Michael Wenz ${ }^{6}$, Philip Muirhead ${ }^{2}$, Nikta Javanfar ${ }^{7}$, and Mario Livio ${ }^{5}$ \\ ${ }^{1}$ Department of Earth and Space Science, UCLA, 595 Charles East Young Drive East, Los Angeles, CA 90095, USA; hilke@ucla.edu \\ 2 Department of Astronomy, California Institute of Technology, MC 130-33, Pasadena, CA 91125, USA \\ ${ }^{3}$ Faculty of Physics, Weizmann Institute of Science, P.O. Box 26, Rehovot 76100, Israel \\ ${ }^{4}$ Racah Institute of Physics, Hebrew University, Jerusalem 91904, Israel \\ ${ }^{5}$ Space Telescope Science Institute, 3700 San Martin Drive, Baltimore, MD 21218, USA \\ ${ }^{6}$ Goddard Space Flight Center, 8800 Greenbelt Road, Greenbelt, MD 20771, USA \\ ${ }^{7}$ Department of Physics, Physics Engineering and Astronomy, Queen's University, 99 University Avenue, Kingston, Ontario K7L 3N6, Canada \\ Received 2012 August 30; accepted 2012 October 29; published 2012 December 4
}

\begin{abstract}
We present here the analysis of about 19,500 new star hours of low ecliptic latitude observations $\left(|b| \leqslant 20^{\circ}\right)$ obtained by the Hubble Space Telescope's Fine Guidance Sensors over a time span of more than nine years, which is in addition to the $\sim 12,000$ star hours previously analyzed by Schlichting et al. Our search for stellar occultations by small Kuiper Belt Objects (KBOs) yielded one new candidate event corresponding to a body with a $530 \pm 70 \mathrm{~m}$ radius at a distance of about 40 AU. Using bootstrap simulations, we estimate a probability of $\approx 5 \%$ that this event is due to random statistical fluctuations within the new data set. Combining this new event with the single KBO occultation reported by Schlichting et al. we arrive at the following results: (1) the ecliptic latitudes of 6.6 and 14.4 of the two events are consistent with the observed inclination distribution of larger, 100-km-sized KBOs. (2) Assuming that small, sub-kilometer-sized KBOs have the same ecliptic latitude distribution as their larger counterparts, we find an ecliptic surface density of KBOs with radii larger than $250 \mathrm{~m}$ of $N(r>250 \mathrm{~m})=1.1_{-0.7}^{+1.5} \times 10^{7} \mathrm{deg}^{-2}$; if sub-kilometer-sized KBOs have instead a uniform ecliptic latitude distribution for $-20^{\circ}<b<20^{\circ}$ then $N(r>250 \mathrm{~m})=4.4_{-2.8}^{+5.8} \times 10^{6} \mathrm{deg}^{-2}$. This is the best measurement of the surface density of sub-kilometer-sized KBOs to date. (3) Assuming the KBO size distribution can be well described by a single power law given by $N(>r) \propto r^{1-q}$, where $N(>r)$ is the number of KBOs with radii greater than $r$, and $q$ is the power-law index, we find $q=3.8 \pm 0.2$ and $q=3.6 \pm 0.2$ for a KBO ecliptic latitude distribution that follows the observed distribution for larger, 100-km-sized KBOs and a uniform KBO ecliptic latitude distribution for $-20^{\circ}<b<20^{\circ}$, respectively. (4) Regardless of the exact power law, our results suggest that small KBOs are numerous enough to satisfy the required supply rate for the Jupiter family comets. (5) We can rule out a single power law below the break with $q>4.0$ at $2 \sigma$, confirming a strong deficit of sub-kilometer-sized KBOs compared to a population extrapolated from objects with $r>45 \mathrm{~km}$. This suggests that small KBOs are undergoing collisional erosion and that the Kuiper Belt is a true analog to the dust producing debris disks observed around other stars.
\end{abstract}

Key words: comets: general - Kuiper Belt: general - methods: observational - occultations - planets and satellites: formation - techniques: photometric

Online-only material: color figures

\section{INTRODUCTION}

The Kuiper Belt consists of a disk of icy objects and is located at the outskirts of our planetary system just beyond the orbit of Neptune. Since the discovery of the first Kuiper Belt Object (KBO) in 1992 (Jewitt et al. 1992), well over 1000 objects have been detected. Dedicated surveys have been revealing an intricate dynamical structure of the Kuiper Belt (e.g., Petit et al. 2011), have been investigating the multiplicity of KBOs (e.g., Noll et al. 2008), and have been measuring the Kuiper Belt size distribution (e.g., Bernstein et al. 2004). In the Kuiper Belt, planet formation never reached completion because runaway growth of the planetary embryos was interrupted, most likely, by an increase in the velocity dispersion of the planetesimals. The size distribution of larger KBOs provides a snapshot of planet formation that was erased elsewhere in the solar system, where planet formation went all the way to completion.

\footnotetext{
8 Hubble Fellow.
}

The cumulative size distribution of KBOs larger than about $45 \mathrm{~km}$ in radius is well described by a single power law given by

$$
N(>r) \propto r^{1-q},
$$

where $N(>r)$ is the number of objects with radii greater than $r$, and $q$ is the power-law index. Observations find that the powerlaw index for large KBOs is $q \sim 4.5$ (Fuentes \& Holman 2008; Fraser et al. 2008). Since this size distribution is a relic of the accretion history in the Kuiper Belt, it provides valuable insights into the formation of large KBOs and the planet formation process itself (e.g., Stern 1996; Davis \& Farinella 1997; Kenyon 2002). Coagulation models of planet formation reproduce the observed size distribution of large KBOs well (e.g., Kenyon \& Luu 1999; Kenyon 2002; Schlichting \& Sari 2011).

Observations suggest that there is a break in the power-law size distribution at smaller KBO sizes (e.g., Bernstein et al. 2004; Fuentes \& Holman 2008; Fraser et al. 2008; Schlichting et al. 2009; Fuentes et al. 2010). The break in the size distribution is generally attributed to collisions that break up small KBOs 
(i.e., $r \lesssim 45 \mathrm{~km}$ ) and subsequently modify their size distribution (e.g., Dohnanyi 1969; Kenyon \& Bromley 2004; Pan \& Sari 2005). If this is so, then the location of the break (i.e., the break radius) constrains the time period over which destructive collisions have been occurring in the Kuiper Belt. This is because with time, collisions move the break radius to ever larger sizes. Furthermore, the power-law index below the break radius constrains the material properties of KBOs. Small KBOs that are in collisional equilibrium and that are held together predominantly by material strength are expected to have a size distribution with power-law index $q=3.5$, which is the so-called Dohnanyi spectrum (Dohnanyi 1969). The size distribution will be shallower with a power-law index of $q \approx 3$ if small KBOs are held together predominantly by gravity (i.e., they are effectively rubble piles; Pan \& Sari 2005). The break radius and the power-law index below the break, therefore, constrain the collisional history of the Kuiper Belt and reveal the material properties of small KBOs, respectively.

Our interest in studying the abundance and size distribution of small $(r<10 \mathrm{~km})$ KBOs is threefold. First of all, we want to determine their overall abundance to test if they are numerous enough to supply the Jupiter Family comets. Second, we want to measure their size distribution in order to constrain collisional evolution in the Kuiper Belt and establish it as a true analog of the dust producing debris disk observed around other stars. And third, we would like to constrain the small KBO size distribution well enough to infer their material properties as discussed above.

Unfortunately, not much is known about the KBO size distribution significantly below the break, because objects with radii less than $\sim 10 \mathrm{~km}$ are too faint to be detected in reflected light. These objects can, however, be detected indirectly by stellar occultations (Bailey 1976; Dyson 1992; Axelrod et al. 1992). A small KBO crossing the line of sight of a star will partially obscure the stellar light, which, under suitable circumstances, can be detected in the star's light curve. Both optical (e.g., Roques et al. 2006; Bickerton et al. 2008; Bianco et al. 2009, 2010; Schlichting et al. 2009; Wang et al. 2010) and X-ray (Chang et al. 2006, 2011) occultation searches have been conducted to probe the population in the Kuiper Belt. Chang et al. (2006) searched for occultation signatures in archival RXTE $\mathrm{X}$-ray data of Scorpius-X1 and reported a surprisingly high event rate. However, Jones et al. (2008) showed that most of the dips in the Scorpius-X1 light curves are artificial effects caused by the RXTE photomultiplier. Only 12 of the original 58 events were not ruled out as artifacts; however, Bickerton et al. (2008) point out that the duration and/or depth of most of the 12 remaining events are inconsistent with the diffraction signature of occultation events. Roques et al. (2006), Bickerton et al. (2008), and Wang et al. (2010) all conducted occultation surveys in the optical regime. None of these surveys reported any detections of objects in the Kuiper Belt, which is not surprising given the low expected event rate of these surveys. Ground-based observations may suffer from a high rate of false positives due to atmospheric scintillation. The only ground-based system that is currently able to screen events caused by atmospheric scintillation and other kinds of interference is the Taiwanese American Occultation Survey (TAOS), which consists of four telescopes that observe the same position simultaneously (e.g., Alcock et al. 2003). TAOS collected over 500,000 star hours with 4 or $5 \mathrm{~Hz}$ sampling frequency and reported no detections so far (Bianco et al. 2010). Recently Doressoundiram et al. (2012) analyzed about 140,000 star hours sampled at $1 \mathrm{HZ}$ by the COROT satellite and reported up to 15 possible $\mathrm{KBO}$ occultation events. The first measurement of the surface density of sub-kilometer-sized KBOs has been reported by Schlichting et al. (2009), who analyzed 12,000 star hours of archival Hubble Space Telescope (HST) data with $40 \mathrm{~Hz}$ sampling frequency. Schlichting et al. (2009) reported one detection and found an ecliptic surface density of KBOs with radii larger than $250 \mathrm{~m}$ of $2.1_{-1.7}^{+4.8} \times 10^{7} \mathrm{deg}^{-2}$.

In this paper, we present the analysis of new data obtained by the Fine Guidance Sensors (FGSs) on board the HST over a time span of more than nine years. We searched this data set for stellar occultations by small KBOs with the aim to measure their abundance to constrain their size distribution and to address the question of whether they are ubiquitous enough to satisfy the supply needed to explain the observed frequency of Jupiter family comets.

This paper is structured as follows. We describe our HSTFGS survey in Section 2. Details about our data analysis and detection efficiency follow in Sections 3 and 4, respectively. In Section 5, we present the results from our survey. We calculate the abundance of sub-kilometer-sized KBOs, compare our findings with other Kuiper Belt surveys, and conclude in Section 6.

\section{THE HST-FGS OCCULTATION SURVEY}

The HST-FGSs are an integral part of the HST pointing control system, ensuring a pointing stability of HST at the milliarcsecond level over exposure times of tens of minutes. Each FGS is a dual-axis white-light shearing interferometer and the photon count of each FGS is recoded by four PMTs (e.g., Fine Guidance Sensor Instrument Handbook: http://www.stsci.edu/hst/ fgs/documents/instrumenthandbook/fgs_ihb.pdf). In addition to ensuring the pointing stability of $H S T$, the FGSs have been used as science instruments. For example, the FGS was used as an astrometric instrument to measure the precise distances to eight galactic Cepheids (Benedict et al. 2007), as an interferometer to resolve binary O-stars in the Carina Nebula (Nelan et al. 2004) and as a high speed photometer to study transit light curves of the planet orbiting HD 17156 and asteroseismology of the star itself (Gilliland et al. 2011).

For over a decade, the three FGSs have been collecting a large number of photometric measurements of the guide stars with $40 \mathrm{~Hz}$ time resolution. In this paper, we search more than nine years of this archival data set for photometric signatures consistent with the occultation of the guide star by a small object in the Kuiper Belt. The $40 \mathrm{~Hz}$ sampling frequency, the large number of star hours of FGS observations that have been obtained over the lifetime of $H S T$, and the stability inherent to space-based observations make this data set ideal for the search of serendipitous stellar occultations by small objects in the outer solar system.

The entire data set, including low and high ecliptic latitude observations and the data analyzed in Schlichting et al. (2009), consists of $\sim 90,000$ star hours. The signal-to-noise ratio $(\mathrm{S} / \mathrm{N})$, defined here as the square root of the mean number of photon counts of all four PMTs combined in a $40 \mathrm{~Hz}$ interval, as a function of star hours is shown in Figure 1 and ranges from about 10 to 100 , reflecting the $9<V<14$ range in magnitude of the HST guide stars. Nominal HST operation uses two FGSs for guiding, with each FGS observing its own guide star. We are therefore able to remove false positives due to instrumental effects and, for example, day/night-time variations due to HST's orbit around the Earth. Figure 2 shows the HST integration time as a function of ecliptic latitude. About $35 \%$ of the 


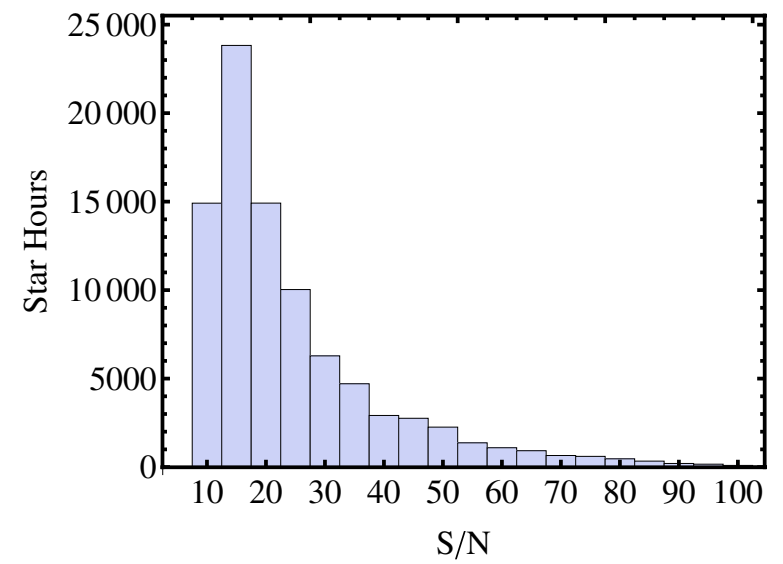

Figure 1. Distribution of star hours as a function of the mean signal-to-noise ratio, $\mathrm{S} / \mathrm{N}$, in a $40 \mathrm{~Hz}$ interval for the entire FGS data set. The $\mathrm{S} / \mathrm{N}$ is defined here as the square root of the mean number of photon counts in a $40 \mathrm{~Hz}$ interval. (A color version of this figure is available in the online journal.)

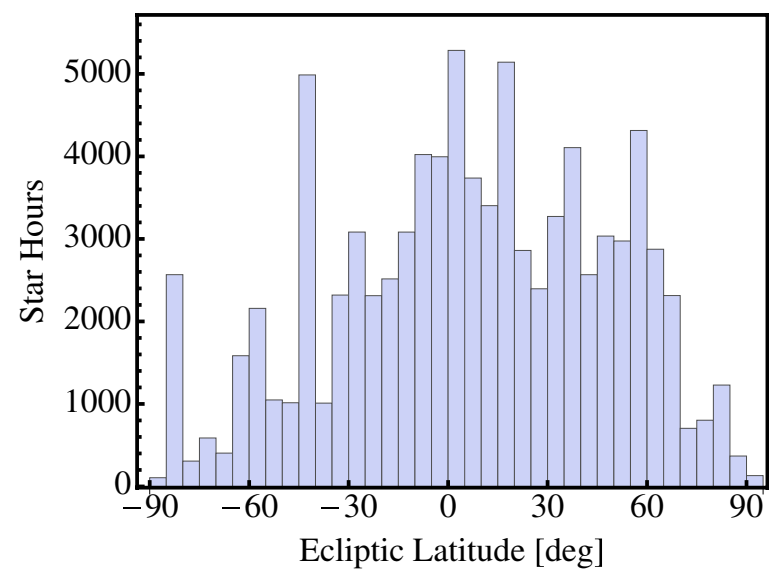

Figure 2. Distribution of star hours as a function of ecliptic latitude for the FGS data set.

(A color version of this figure is available in the online journal.)

observations are taken in the low ecliptic latitude region (i.e., $-20^{\circ}<i<+20^{\circ}$ ) of the Kuiper Belt. The remaining high ecliptic latitude observations provide an excellent control sample which we use to test for possible false positives. Figure 3 shows the distribution of angular sizes of the HST guide stars in our data set in units of the Fresnel angular scale at $40 \mathrm{AU}$ and for observations at a wavelength of $600 \mathrm{~nm}$. The Fresnel angular scale is given by $\sim \sqrt{\lambda / 2 a}$, where $\lambda$ is the wavelength of the observations and $a$ is the semimajor axis of the Kuiper Belt. About $63 \%$ of the stars in our data set subtend angular sizes less than 0.5 Fresnel angular scales at a distance of $40 \mathrm{AU}$, and $84 \%$ of all stars in our data set have angular sizes of less than 1 Fresnel scale. The diffraction pattern that is produced by a sub-kilometer-sized $\mathrm{KBO}$ occulting an extended background star is smoothed over the finite stellar disk. This effect becomes clearly noticeable for stars that subtend sizes larger than about 0.5 Fresnel scales and reduces the detectability of occultation events around such stars. The distribution of the finite angular sizes of the stars is taken into account when we calculate the detection efficiency of our survey in Section 4.

Finally, we analyzed the entire data set for correlated noise by calculating the autocorrelation function with lags of $0.025 \mathrm{~s}$. Most of the data sets, each consisting of the observations obtained over one $H S T$ orbit, are free of statistically significant

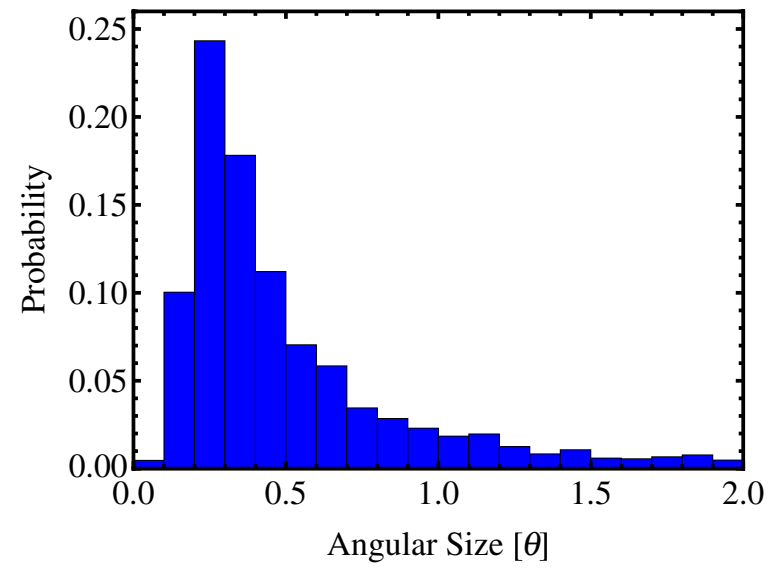

Figure 3. Distribution of angular sizes of the HST guide stars in our data set, in units of the angular Fresnel scale. The angular sizes of the guide stars were calculated by fitting 2MASS JHK and USNO-B1 BR photometry with a blackbody spectrum.

(A color version of this figure is available in the online journal.)

correlated noise. About $17 \%$ of the roughly 120,000 the data sets contained correlated noise, which is significant at, or above, the $4.5 \sigma$ level for a given data set and significant at, or above, the $1 \sigma$ level for the analysis of the entire data, which consisted of 120,000 independent trials. This correlated noise is often due to slopes, i.e., long-term variability, in the data. Such long-term trends may affect the results from a bootstrap analysis, however, we show that the two data files containing candidate events for which we use a bootstrap analysis to estimate their significance do not have any statistically significant correlated noise (see Section 5 for details).

\section{DATA ANALYSIS}

The $40 \mathrm{~Hz}$ time resolution of our survey allows for the detection of the actual diffraction pattern rather than a simple decrease in the photon counts. Our detection algorithm therefore employs a template search using theoretical light curves and performs $\chi^{2}$ fitting of the templates to the data. This template fitting procedure improves the sensitivity of the survey compared to algorithms that search only for dips in the light curve and aids with the identification of false positives.

In general, the occultation diffraction pattern is determined by the size of the KBO, the angular size of the star, the wavelength range of the observations, and the impact parameter between the star and the KBO. The typical KBO sizes that our survey is most likely to detect is determined by the $\mathrm{S} / \mathrm{N}$ of our data, because small KBOs are more numerous than their larger counterparts and the amplitude of the occultation signal scales as the crosssection of the KBO. Given a typical S/N of 15 in a $40 \mathrm{~Hz}$ interval (see Figure 1), our survey is most likely to detect KBO occultation events caused by objects that are a few hundred meters in radius. Since these objects are significantly smaller than the Fresnel scale, they give rise to occultation events that are in the Fraunhofer diffraction regime. This implies that the diffraction pattern itself is not sensitive to the exact shape of the $\mathrm{KBO}$, but the amplitude of the diffraction pattern scales linearly as the cross section of the occulter. This significantly reduces the number of templates that need to be implemented in the search algorithm. Furthermore, the templates that we use in our search algorithm treat the stars as point sources. The reason for this is twofold. First, a large fraction $(\sim 63 \%)$ of the stars in our survey have angular sizes below 0.5 Fresnel scales, which 
implies that the point source template is a good approximation for these stars. Second, using templates with finite angular size stars hurts rather than helps the detection efficiency because the typical uncertainties in the estimated angular radii are too large (i.e., they are typically about $40 \%$ ). Since the diffraction pattern is wavelength dependent, we integrate the light curve templates that we use in our detection algorithm over a wavelength range of the FGS observations which extends from 400 to $700 \mathrm{~nm}$. Finally, our search algorithm includes light curve templates for impact parameters between the KBO and background star ranging from 0 to 1.0 Fresnel scales in steps of 0.2 Fresnel scales.

For a given impact parameter, our theoretical light curves have three free parameters. The first is the mean number of photon counts, $m$, which is the normalization of the light curve. The second is the amplitude of the occultation, $A$, which is proportional to the cross section of the $\mathrm{KBO}$, and the third is the duration of the occultation, which is inversely proportional to the relative velocities between $H S T$ and the KBO. We obtain values for the first two parameters from our data by minimizing $\chi^{2}$ where

$$
\chi^{2}=\sum_{i=1}^{n} \frac{\left(d_{i}-\operatorname{model}_{i}\right)^{2}}{d_{i}}
$$

and

$$
\operatorname{model}_{i}=m\left(A\left(l_{i}-1\right)+1\right),
$$

where $l_{i}$ is our theoretical light curve template. We can simply solve for the values of $A$ and $m$ from our data and therefore only need to perform a full search for the third free parameter, which is the duration of the occultation and time. The duration of the occultation is independent of the object size, and mainly determined by the ratio of the Fresnel scale to the relative speed between HST and the KBO perpendicular to the line of sight. This relative speed is given by the combination of HST's velocity around the Earth, Earth's velocity around the Sun, and the velocity of the KBO itself. We use this information to restrict the parameter space for the template widths in our search such that we are sensitive to KBOs located at the distance of the Kuiper Belt between $30 \mathrm{AU}$ and $60 \mathrm{AU}$, but allow for $\mathrm{KBO}$ random velocities of up to $v_{\text {Kepler }}=5 \mathrm{~km} \mathrm{~s}^{-1}$.

Our search algorithm identifies occultation candidates by calculating their $\Delta \chi^{2}$, which we define as the difference between the $\chi^{2}$ value calculated for a horizontal line, corresponding to no event, and the $\chi^{2}$ value derived from best-fit light curve template. Occultation events have large $\Delta \chi^{2}$ since they are poorly fitted by a constant straight line, but well matched by the light curve template. If the noise properties were identical over the entire data set, then the probability that a given occultation candidate is due to random noise can be characterized by a single value of $\Delta \chi^{2}$ for all observations. In reality, however, the noise properties are different from observation to observation; non-Poisson especially tails in the photon counts distribution will give rise to slightly different $\Delta \chi^{2}$ distributions. Therefore, ideally, we would determine a unique detection criterion for each individual HST orbit. However, this would require us to simulate each data set, which contains about an hour of observations in a single HST orbit, over the entire length of our survey, which is not feasible due to the enormous computational resources that would be required. Instead, we perform bootstrap simulations over all the FGS data sets together and use this to estimate the typical $\Delta \chi^{2}$ value that we use in our detection algorithm (Efron 1982). Using bootstrap simulations for estimating the significance of candidate events is justified as long as there is no

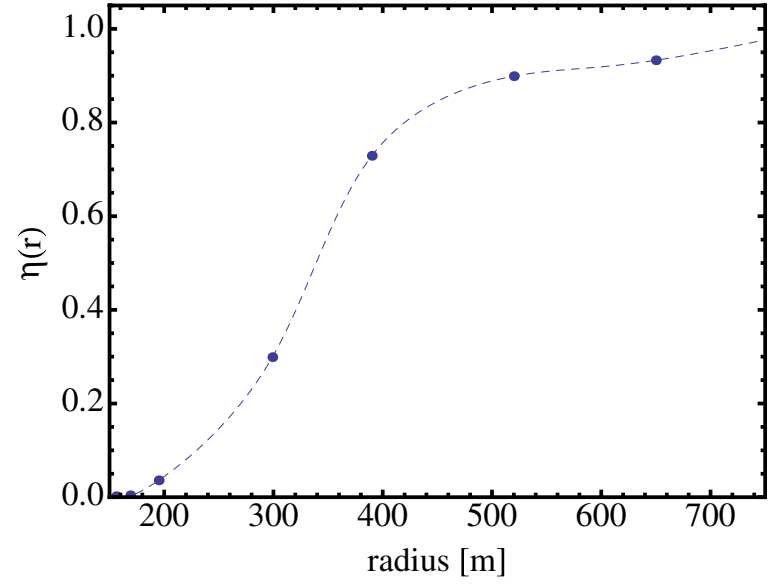

Figure 4. Detection efficiency, $\eta(r)$, as a function of KBO radius. The detection efficiency is normalized by an impact parameter equal to 1 Fresnel scale, i.e., a detection efficiency of 1 means that the effective cross-section for detection has a radius equal to 1 Fresnel scale. The detection efficiency of our survey is $\sim 4 \%$ for objects with $r=200 \mathrm{~m}$ and $75 \%$ for KBOs with $r=400 \mathrm{~m}$.

(A color version of this figure is available in the online journal.)

correlated noise in the data. For all occultation candidates that exceed this detection threshold, we determined their statistical significance, i.e., the probability that they are due to random noise, by extensive bootstrap simulations of the individual data sets. Our detection algorithm flagged all events for which the template fit of the diffraction pattern was better than $15 \sigma$ and that had a $\Delta \chi^{2}>63$. This detection criterion corresponds to about 0.5 false-positive detections over the 19,500 new star hours of low ecliptic latitude observations that are analyzed in this paper. All flagged events that were solely due to one single low or high point were ignored in the data analysis, but included as false positives when calculating the significance of candidate occultation events. This eliminates a large number of otherwise flagged events that may, for example, be caused by cosmic rays.

\section{DETECTION EFFICIENCY}

The ability to detect an occultation event of a given size $\mathrm{KBO}$ depends on the impact parameter of the $\mathrm{KBO}$, the duration of the event, the angular size of the star, and the $\mathrm{S} / \mathrm{N}$ of the data. We calculate the detection efficiency of our survey by planting synthetic events with different radii into the FGS data and analyzing this modified data set with the same search algorithm that we used to analyze the original FGS data with the same significance threshold of $\Delta \chi^{2}>63$. The synthetic events correspond to $\mathrm{KBO}$ sizes ranging from $200 \mathrm{~m}<r<850 \mathrm{~m}$, they have impact parameters from 0 to 2.5 Fresnel scales and a relative velocity distribution that is identical to that of the actual FGS observations. To account for the finite angular sizes of the stars we generated light curve templates with stellar angular radii of $0.1,0.3,0.4,0.6,0.8$, and 1 Fresnel scales. The detection efficiency of our survey was calculated using the angular size distribution of the FGS guide stars as shown in Figure 3 and the synthetic events were implanted in a subset of the FGS data that had the same $\mathrm{S} / \mathrm{N}$ properties as the whole data set shown in Figure 1. Figure 4 shows the detection efficiency, $\eta(r)$, as a function of $\mathrm{KBO}$ radius. We normalize our detection efficiency for a given size KBO to 1 for an effective detection cross section with a radius of 1 Fresnel scale. The detection efficiency of our survey is $\sim 0.04$ for objects with $r=200 \mathrm{~m}$ and $\sim 0.75$ for KBOs with $r=400 \mathrm{~m}$ located at $40 \mathrm{AU}$. 


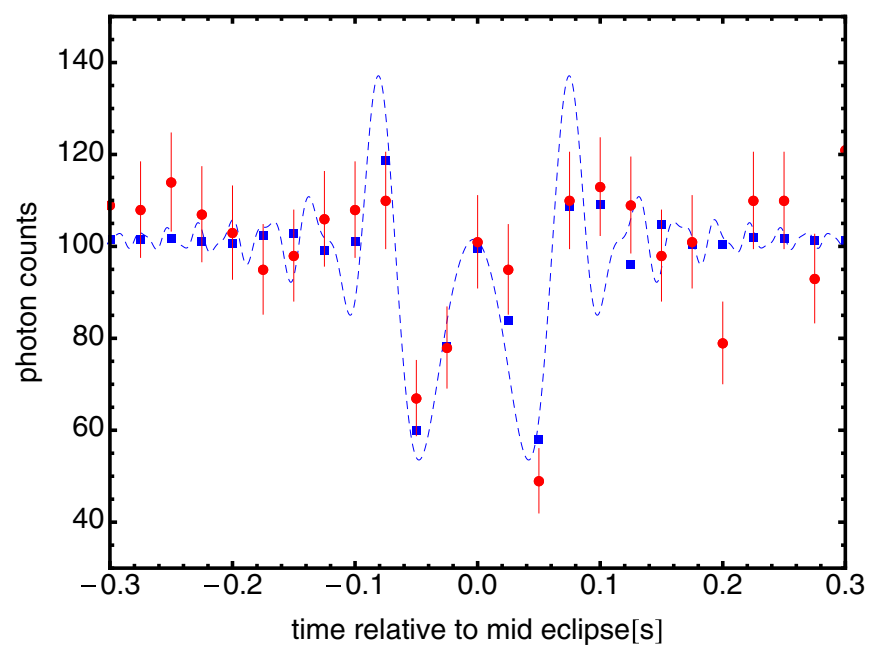

Figure 5. Photon counts as a function of time of the candidate occultation event observed by FGS3. The red points and error bars are the FGS data points with Poisson error bars, the dashed blue line is the theoretical light curve, and the blue squares correspond to the theoretical light curve template in our detection algorithm integrated over $40 \mathrm{~Hz}$ intervals. We note here that the actual noise for this observation is about $8 \%$ larger than poison noise, due to additional noise sources such as, for example, dark counts, which contribute about 3-6 counts per PMT in a $40 \mathrm{~Hz}$ Interval. The best-fit $\chi^{2} /$ dof from our detection algorithm is $27.3 / 28$. The star has an ecliptic latitude of +6.6 and its angular radius and effective temperature are $0.58 \pm 0.06$ Fresnel scales and $\sim 5000 \mathrm{~K}$, respectively. The position of the star is R.A. $=64.74065$, decl. $=28.13064(\mathrm{~J} 2000)$, and its estimated $V$ magnitude is 13.9. Assuming a circular orbit, the best-fit parameters yield a KBO size of $r=530 \pm 70 \mathrm{~m}$ and a distance of $35 \pm 9$ AU.

(A color version of this figure is available in the online journal.)

\section{RESULTS}

\subsection{One New Occultation Candidate}

Among the $\sim 40$ candidate events that were flagged with $\Delta \chi^{2}>63$ in the new data set, all but one turned out to be false positives (see Section 5.2 for a detailed discussion of the false positives), leaving us with one new occultation candidate event, shown in Figures 5 and 6. Figure 5 shows the candidate event with the best-fit template from our search algorithm. The red points and error bars represent the FGS data with Poisson error bars. The actual noise in this observation is about $8 \%$ larger than the Poisson noise, which is due to additional noise sources such as dark counts, which contribute about 3-6 counts for a given PMT in a $40 \mathrm{~Hz}$ interval. The mean $\mathrm{S} / \mathrm{N}$ in a $40 \mathrm{~Hz}$ interval for this $H S T$ orbit of observations is $\sim 10$. The best-fit $\chi^{2} /$ dof from our detection algorithm is $27.3 / 28$. Each FGS provides two independent PMT readings and we confirmed that the occultation signature is present in both of the these independent photon counts. The position of the star is R.A. = 64.74065 , decl. $=28.13064(\mathrm{~J} 2000)$, which translates to an ecliptic latitude of +6.6 . We obtained a spectrum of this star on 2012 February 12 with the Echelle Spectrograph and Imager (ESI) on the Keck II telescope (Sheinis et al. 2002). We used ESI's echellete mode with a 0'.5 slit width, providing spectral coverage from 3900 to $11000 \AA$ simultaneously with a resolving power $(\lambda / \Delta \lambda)$ of 8100 . We exposed for $300 \mathrm{~s}$, achieving a median $\mathrm{S} / \mathrm{N}$ of 75 between 6000 and $9000 \AA$. Analysis of the stellar spectrum and fitting the $J H K$ bands from Two Micron All Sky Survey (2MASS; Skrutskie et al. 2006) yields an stellar angular radius and effective temperature of $0.58 \pm 0.06$ Fresnel scales and $\approx 5000 \mathrm{~K}$, respectively. Using our best estimate for the stellar angular radius, we find that the best-fit parameters yield a KBO size of $r=530 \pm 70 \mathrm{~m}$ and, assuming a circular orbit, a

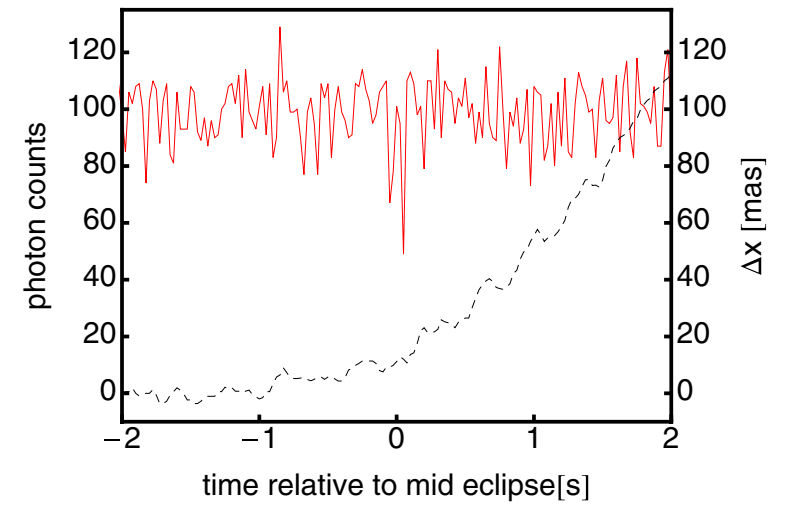

Figure 6. Photon counts as a function of time during the occultation event observed by FGS3. The red-solid line represents the $40 \mathrm{~Hz}$ photon counts from FGS3, while the black dashed line shows the displacement of the guide star along the $x$-axis, $\Delta x$, in the FGS relative to its position at time $=-2 \mathrm{~s}$. The occultation event occurred (at time $=0$ ) approximately one second after HST began a commanded small angle maneuver associated with a planned displacement of the active science instrument's aperture on the sky. During this maneuver FGS3 actively tracked its guide star, keeping it at interferometric null, just as it did prior to the maneuver. Thus, the decrease of photon counts near time $=0$ is not correlated with or caused by the telescope repointing. Note that the displacement along the FGS $y$-axis was far smaller and is therefore not plotted here.

(A color version of this figure is available in the online journal.)

distance of $35 \pm 9$ AU. We note here, that within the uncertainties of the actual stellar angular radius, that light curve templates with smaller angular radii give a somewhat better fit to the data than templates with larger stellar radii. For objects on circular orbits around the Sun, two solutions can fit the duration of the event. However, the second solution corresponds to a distance of $0.2 \mathrm{AU}$ from the Earth and an object size of $\approx 50 \mathrm{~m}$, and is therefore unlikely. It is also unlikely that the occulting object was located in the Asteroid belt, since the expected occultation rate from Asteroids is about two orders of magnitude less than our implied rate. In addition, an Asteroid would have to have an eccentricity of 0.2 or greater to match the duration of the observed occultation candidate.

We estimate the probability that this candidate event is due to statistical fluctuations using bootstrap simulations. This approach is justified as long as there is no correlated noise in the data. We calculate the autocorrelation for lags between 0 and $1 \mathrm{~s}$ for the HST orbit of observations that contained the event and find that the autocorrelation function is consistent with zero (see Figure 7). Using the data from the HST orbit, which contained the event, we removed the event itself and simulated $3.1 \times 10^{6}$ star hours, which corresponds to 161 times the low ecliptic latitude observations analyzed in this paper. This calculation required $\sim 2300$ CPU days of computing power. Figure 8 shows the cumulative number of false positives, $N_{f-p}$ as a function of $\Delta \chi^{2}$. The number of false positives was normalized to 19,500 star hours, which correspond to the length of the entire low ecliptic latitude observations analyzed here. In the entire bootstrap analysis we obtained eight events with a $\Delta \chi^{2} \geqslant 71.9$. This implies a probability of $\sim 5 \%$ that events like the occultation candidate with $\Delta \chi^{2}=71.9$ are caused by random statistical fluctuations over the entire low ecliptic latitude observations analyzed in this paper.

\subsection{False Positives}

Among the $\sim 40$ candidate events that were flagged because they had a $\Delta \chi^{2}>63$, all but one turned out to be false positives. 


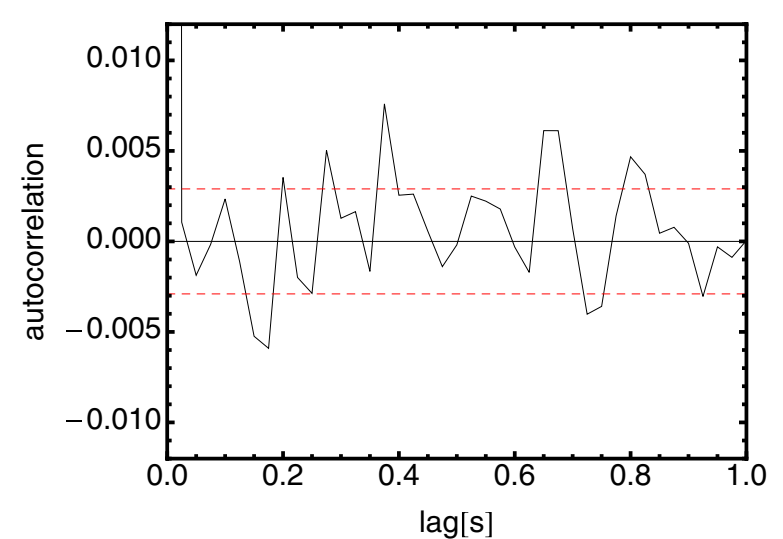

Figure 7. Autocorrelation function for lags between 0 and $1 \mathrm{~s}$ for the $H S T$ orbit of observations containing the candidate event shown in Figure 5. We note that the autocorrelation at lag 0 is, by definition, equal to one. The red-dotted lines show the upper and lower $1 \sigma$ errors of the autocorrelation. The autocorrelation function of this data is consistent with zero.

(A color version of this figure is available in the online journal.)

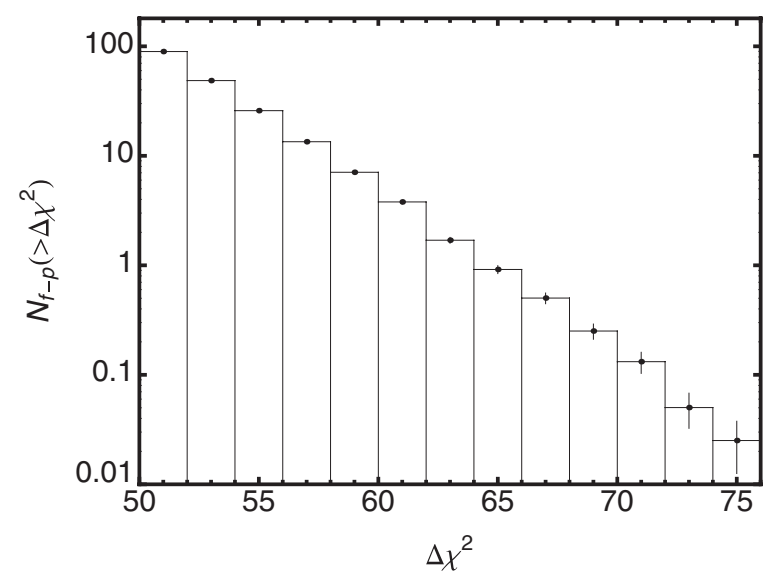

Figure 8. Cumulative number of false positives, $N_{f-p}$, as a function of $\Delta \chi^{2}$ for the candidate event normalized to 19,500 star hours, which correspond to the length of the entire low ecliptic latitude observations analyzed in this paper. The false positives were obtained from bootstrap simulations using the one orbit of $H S T$ observations ( $\sim 50$ minutes) in which we found the candidate event. From these bootstrap simulations, we find a probability of $\approx 5 \%$ that events like the occultation candidate with $\Delta \chi^{2}=71.9$ are caused by random statistical fluctuations in the entire low ecliptic latitude data set.

The most common false positives were due to what looks like a slower read out of the photon counts (see Figure 9) or showed a correlation between the signature in the photon counts and the displacement of the guide star from its null position on the FGS (see Figure 10). Figure 10 shows an example of a false positive that shows a strong correlation between the number of photon counts and the displacement of the guide star from its null position on the FGS. The jitter introduced due to the displacement of the guide star from its null position causes an up to $3 \%$ change in the photon counts and is therefore only detected by our search algorithm for stars that have photon counts above a few thousand in a single $40 \mathrm{~Hz}$ interval. The jitter due to the guide star's displacement has a characteristic frequency of $1 \mathrm{~Hz}$ and is therefore easy to identify.

\subsection{High-ecliptic Latitude Control Sample}

In addition to the 19,500 star hours of low ecliptic latitude observations, $|b|<20^{\circ}$, we also analyzed $\sim 36,000$ star hours of high ecliptic latitude, $|b|>20^{\circ}$, observations and used these

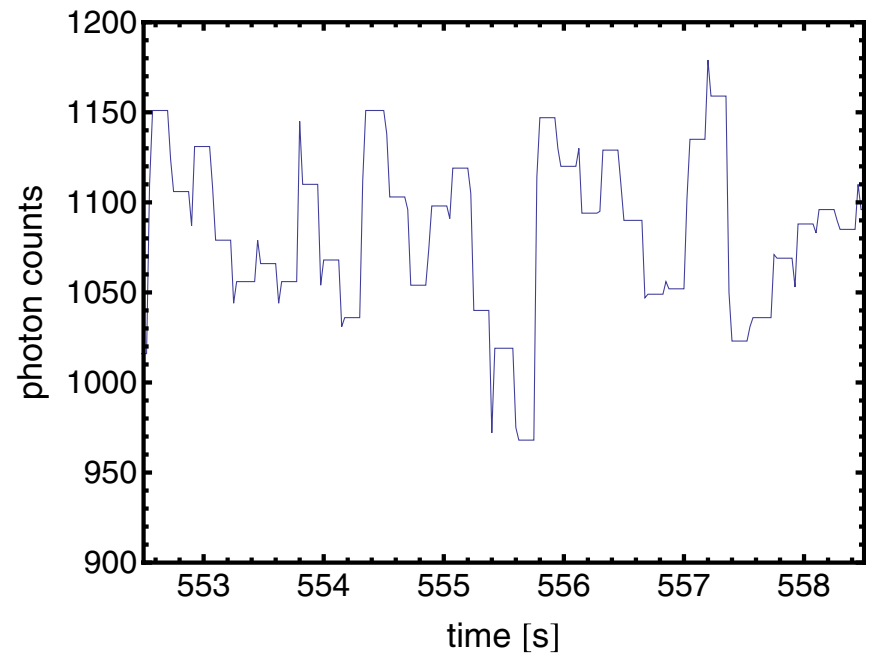

Figure 9. Photon counts as a function of time. An example of a false positive found in the FGS data, which is due to what looks like a slower read out of the photon counts. False positives like this one are due to update problems that are encountered as the telemetry from $H S T$ goes through various transfer stations and ground stations on its way to the archive.

(A color version of this figure is available in the online journal.)

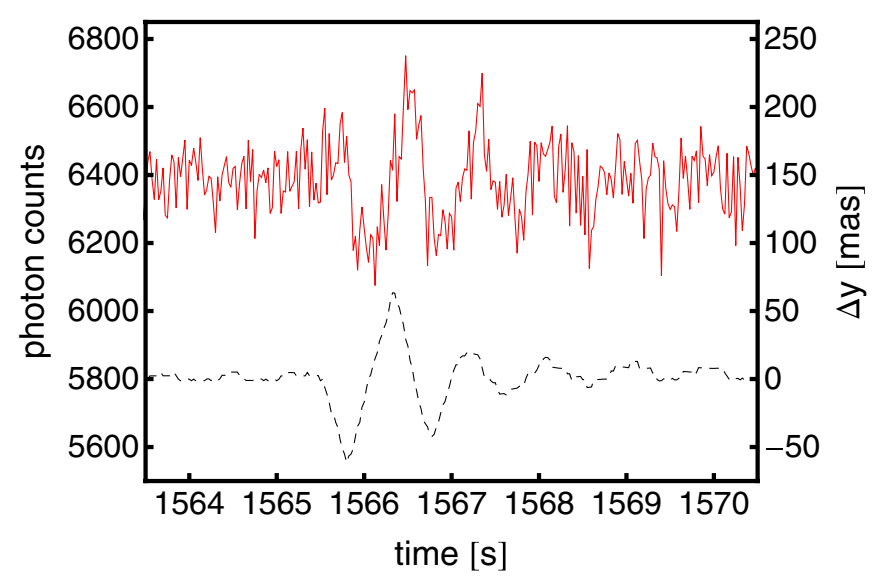

Figure 10. Example of a false positive that is due to jitter introduced by the displacement of the guide star from its null position. The red solid line represents the photon counts of the FGS data and the black dashed line the corresponding displacement of the guide star relative to its null position along the $y$-axis, as recorded by the FGS, as a function of time.

(A color version of this figure is available in the online journal.)

as a control sample. We analyzed the high ecliptic latitude observations with exactly the same detection algorithm as the low ecliptic latitude data. A total of $\sim 70$ candidate events were flagged with $\Delta \chi^{2}>63$. All but one of these events were due to either jitter, induced due to the displacement of the guide star from its null position (see Figure 10), or a lower read out frequency of the PMTs (see Figure 9). The only event which was not caused by either of these two effects is shown in Figure 11. The ecliptic latitude of this event is 81.5 and it has a $\Delta \chi^{2}=72.3$. Calculation of the autocorrelation function of this orbit of $H S T$ observations showed that there is no statistical significant correlated noise in the data. Using bootstrap simulations we find a $21 \%$ probability that this event is due to random statistical fluctuations (see Figure 12), given the entire high ecliptic latitude data set analyzed in this paper. This implies a $\sim 79 \%$ chance that this flagged event is due to a high inclination $\mathrm{KBO}$, but 


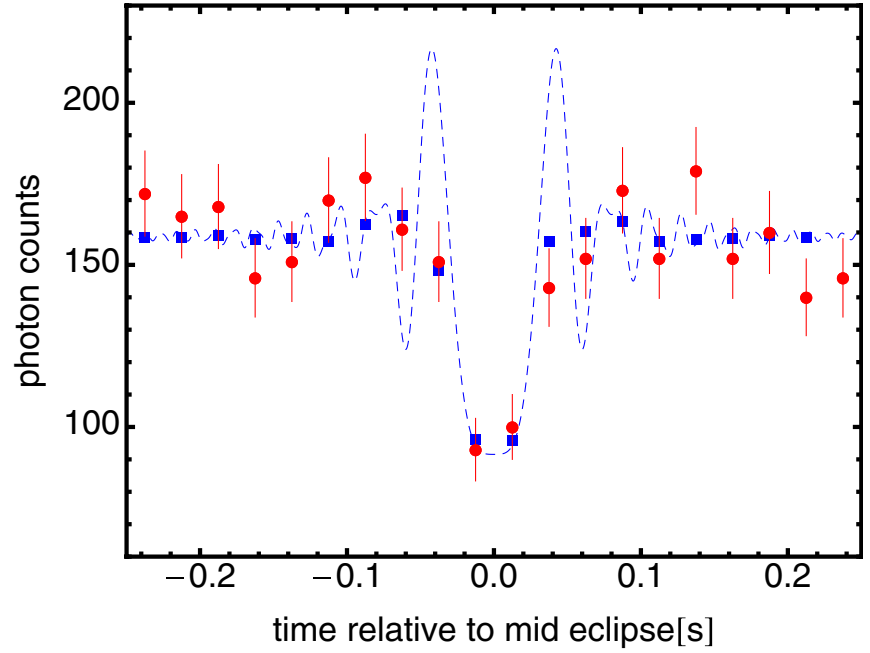

Figure 11. Photon counts as a function of time of the only flagged event in our control sample that could not be attributed to either jitter, induced due to the displacement of the guide star from its null position (see Figure 10), or a lower read out frequency of the PMTs (see Figure 9). The red points and error bars are the FGS data points with Poisson error bars, the dashed blue line is the theoretical light curve, and the blue squares correspond to the theoretical light curve integrated over $40 \mathrm{~Hz}$ intervals. The best-fit $\chi^{2} /$ dof is $13.5 / 17$. Bootstrap simulations yield a probability of $\sim 21 \%$ that events like this with $\Delta \chi^{2}=72.3$ are caused by random statistical fluctuations over the high ecliptic latitude control sample analyzed in this paper. This implies a $\sim 79 \%$ chance that this flagged event is due to a high inclination $\mathrm{KBO}$, but this interpretation seems unlikely given the known inclination distribution of large KBOs.

(A color version of this figure is available in the online journal.)

this interpretation seems unlikely given the known inclination distribution of large KBOs.

\section{DISCUSSION AND CONCLUSIONS}

We combine the one candidate KBO occultation event presented in this paper, which we found in 19,500 star hours of low ecliptic latitude HST-FGS observations, with the single event that was reported by Schlichting et al. (2009). Schlichting et al. (2009) analyzed 12,000 star hours of low ecliptic latitude HSTFGS observations and reported one KBO occultation event at an ecliptic latitude of +14 . 4 . First, we test whether the ecliptic latitudes of the two events are consistent with the observed inclination distribution of larger KBOs and then we use the two events to estimate the abundance of sub-kilometer-sized KBOs.

\subsection{Inclination Distribution}

In this subsection, we test if the observed ecliptic latitude distribution of the two occultation events is consistent with the inclination distribution of larger KBOs inferred from direct searches.

We first calculate for each star in our data set the ecliptic latitude, and the amount of time it was observed by the FGSs. Figure 2 shows the distribution of star hours as a function of ecliptic latitude. We denote this function by $t_{\mathrm{FGS}}(\beta)$. Using the inclination distribution of KBOs, $P\left(i_{\mathrm{KBO}}\right)$, from Elliot et al. (2005), we randomly draw inclinations from $P\left(i_{\mathrm{KBO}}\right)$ and for each random declination we choose a random ecliptic latitude $\sin (\beta)=\sin (i) \sin (\lambda) .{ }^{9}$ Here the ecliptic longitude, $\lambda$, is a random number drawn from a uniform distribution between 0 and $2 \pi$. This give us the probability distribution of the

\footnotetext{
9 Specifically, we use the numerical inclination distribution presented in Figure 20 of Elliot et al. (2005).
}

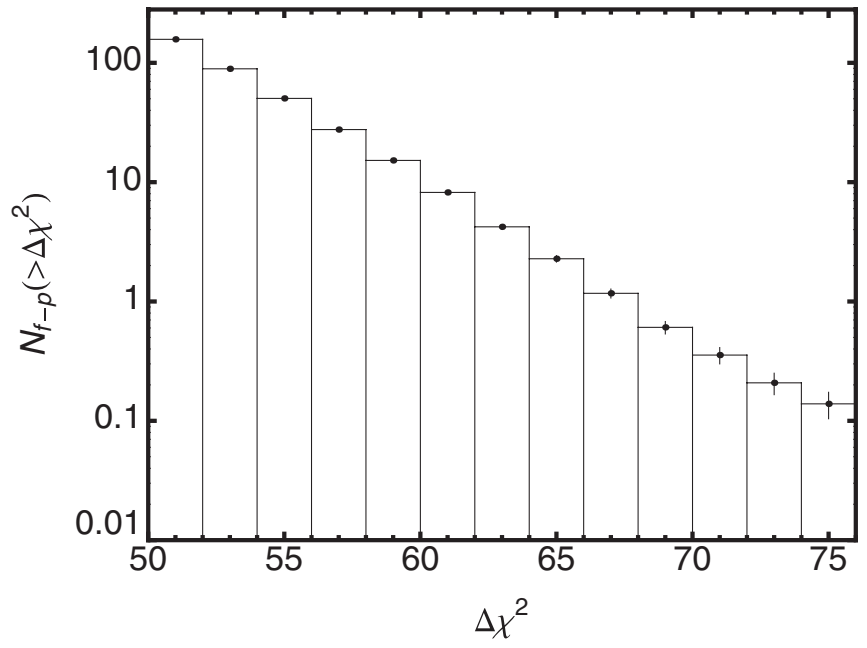

Figure 12. Cumulative number of false positives, $N_{f-p}$, as a function of $\Delta \chi^{2}$ for the flagged event found in the high ecliptic latitude control sample. The number of false positives is normalized to 36,000 star hours, which correspond to the length of the entire high ecliptic latitude observations analyzed in this paper. The false positives plotted here were obtained from bootstrap simulations using the one orbit of HST observations in which this event occurred. From these bootstrap simulations, we find a probability of $\sim 21 \%$ that events like this with $\Delta \chi^{2}=72.3$ are caused by random statistical fluctuations over the high ecliptic latitude control sample analyzed in this paper.

instantaneous ecliptic latitude distribution, $P_{\mathrm{KBO}}(\beta)$, associated with inclination distribution from Elliot et al. (2005). Figure 13 shows the product of $P_{\mathrm{KBO}}(\beta)$ and $t_{\mathrm{FGS}}(\beta)$ (denoted by $P_{\mathrm{FGS}}(\beta)$ ), and gives the probability that our survey will detect a KBO at a given ecliptic latitude, assuming the KBOs are drawn from the Elliot et al. (2005) inclination distribution. We assumed here that KBOs follow circular orbits and that the $\mathrm{S} / \mathrm{N}$ distribution of the FGS data is the same at all ecliptic latitudes.

In order to see if our observations are consistent with the inclination distribution of large KBOs, we calculate the probability distribution for observing two events at different latitudes using the detection probability as a function of ecliptic latitude shown in Figure 13. The log-probability of our ecliptic latitude observations is: $\sum_{j}^{2} \ln P_{\mathrm{FGS}}\left(\beta_{\mathrm{j}}\right)$, where $\beta_{\mathrm{j}}$ are our observed ecliptic latitudes of +6.6 and +14 . 4 . In order to put this into context, we need to estimate the log-probability distribution given our data set. This is done using Monte Carlo simulations, which consist of drawing 10,000 random pairs of ecliptic latitudes and calculating the log-probability for each pair. Figure 14 shows the probability distribution, where the arrow denotes the value of the log-probability of our observations. This plot suggests that our observed ecliptic latitudes are consistent with ecliptic latitudes drawn from the inclination distribution of large KBOs. However, this conclusion would change if the flagged event in our control sample (discussed in Section 5.3) was due to an actual KBO.

\subsection{Abundance of Sub-kilometer-sized KBOs}

We now combine the results from this paper with the work of Schlichting et al. (2009) to estimate the abundance of subkilometer-sized KBOs.

The number of occultation events is given by

$$
N_{\mathrm{events}}=-2 v_{\mathrm{rel}} F \int_{r_{\min }}^{r_{\max }} \int_{-b}^{b} \eta(r) \frac{\Delta t}{\Delta b} \frac{d N(r, b)}{d r} d b d r
$$




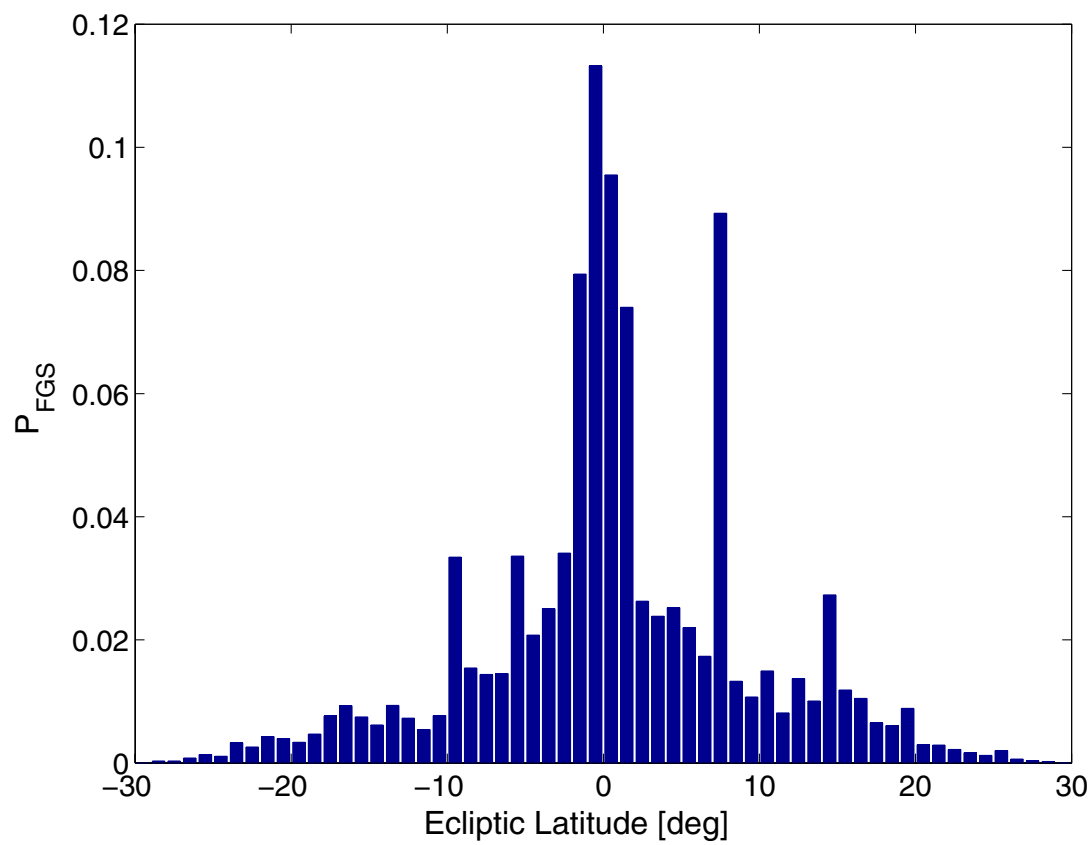

Figure 13. Occultation detection probability per degree, $P_{\mathrm{FGS}}$, as a function of ecliptic latitude for the low ecliptic latitude observations $|b|<20^{\circ}$ of the FGS data set. The detection probability was calculated from the ecliptic latitude distribution of the FGS data shown in Figure 2. Note that we assumed that the KBO ecliptic latitude distribution is symmetric about the ecliptic and ignored the small 1.6 inclination of the Kuiper Belt plane relative to the ecliptic.

(A color version of this figure is available in the online journal.)

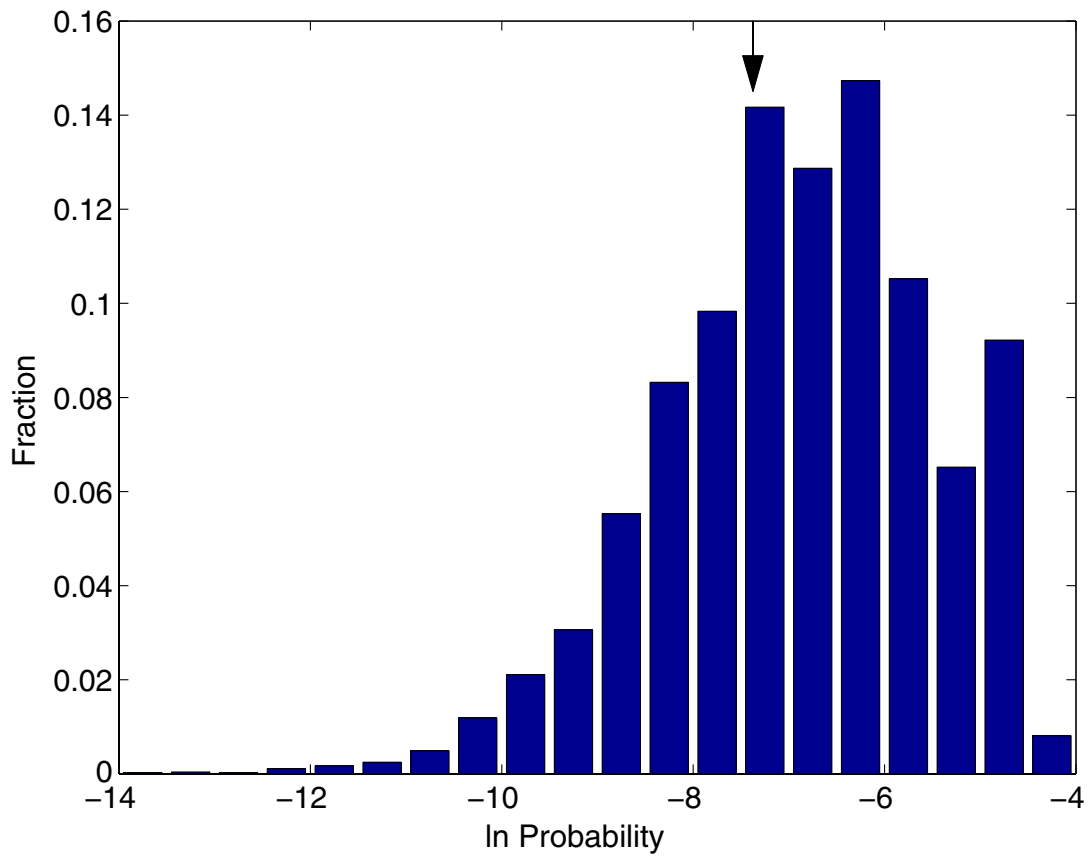

Figure 14. Probability distribution derived from Monte Carlo simulations, which consisted of drawing 10,000 random pairs of ecliptic latitudes from the distribution shown in Figure 13 and calculating the probability for each pair. The arrow denotes the log-probability value of the of our observations, suggesting that our observed ecliptic latitudes are consistent with ecliptic latitudes drawn from the inclination distribution of large KBOs.

(A color version of this figure is available in the online journal.)

where $v_{\text {rel }}=22 \mathrm{~km} \mathrm{~s}^{-1}$ is the typical relative velocity between the $\mathrm{KBO}$ and the observer, $b$ is the ecliptic latitude, $\Delta t / \Delta b$ is the time observed per degree in ecliptic latitude, as shown in Figure 2, and $F=1.3 \mathrm{~km}$ is the Fresnel scale. The sky density of KBOs is both a function of ecliptic latitude, $b$, and the KBO radius, $r$. Therefore, in order to estimate the total number of $\mathrm{KBOs}$ of a given size or their corresponding sky density, we need to make an assumption regarding their ecliptic latitude distribution. Unfortunately, very little is currently know about the ecliptic latitude distribution of sub-kilometer-sized objects in the Kuiper Belt. We therefore estimate the total number of KBOs for two very different ecliptic latitude distributions, currently both are consistent with the ecliptic latitudes of the candidate event presented in this paper and the event reported by Schlichting et al. (2009). In the first case, we assume that the $\mathrm{KBO}$ latitude distribution, $f(b)$, is uniform 
between $-20^{\circ}$ and $+20^{\circ}$, such that $f(b)=1$ for $-20^{\circ}<$ $b<20^{\circ}$ and zero otherwise. In the second case we assume that small sub-kilometer-sized KBOs follow the same ecliptic latitude distribution as their larger 100-km-sized counterparts and use the ecliptic latitude distribution provided in Elliot et al. (2005) in Figure 14. We further assume that the KBO size distribution follows a power law, such that it can be written as $N(r, b)=n_{0} r^{-q+1} f(b)$, where $n_{0}$ is the normalization factor for the cumulative surface density of KBOs. Substituting for $d N(r, b) / d r$ in Equation (4) and solving for $n_{0}$ we get

$$
n_{0}=\frac{N_{\text {events }}}{2 v_{\text {rel }} F(q-1) \int_{r_{\min }}^{r_{\max }} \eta(r) r^{-q} d r \int_{-b}^{b} f(b) \frac{\Delta t}{\Delta b} d b} .
$$

Evaluating Equation (5) assuming a uniform KBO ecliptic latitude distribution for $-20^{\circ}<b<20^{\circ}$ yields a cumulative KBO surface density of

$$
N(r>250 m)=4.4_{-2.8}^{+5.8} \times 10^{6} \mathrm{deg}^{-2} .
$$

Similarly, evaluating Equation (5), assuming that the small, subkilometer-sized KBOs follow the ecliptic latitude distribution from Elliot et al. (2005), yields a cumulative KBO surface density averaged over the ecliptic $\left(|b|<5^{\circ}\right)$ of

$$
N(r>250 m)=1.1_{-0.7}^{+1.5} \times 10^{7} \mathrm{deg}^{-2} .
$$

When evaluating the integral over $r$ in Equation (5), we assumed $q=4$. We note however that the value for the cumulative KBO surface density at $r=250 \mathrm{~m}$ only weakly depends on the exact choice for $q$. We therefore find an ecliptic KBO abundance for bodies with $r>250 \mathrm{~m}$ that ranges, depending on the actual inclination distribution of sub-kilometer-sized KBOs, between $4.4 \times 10^{6} \mathrm{deg}^{-2}$ to $1.1 \times 10^{7} \mathrm{deg}^{-2}$. This is the best measurement of the surface density of sub-kilometer-sized KBOs to date and about a factor of two lower than the first results published by Schlichting et al. (2009). Figure 15 displays the results from the FGS survey and summarizes published upper limits from various works. The red point plotted at $r=250 \mathrm{~m}$ with the upper and lower error bars in Figure 15 gives the best estimate of the KBO surface density around the ecliptic $\left(-5^{\circ}<b<5^{\circ}\right)$ from our survey with $1 \sigma$ errors, assuming that sub-kilometersized KBOs follow the same inclination distribution as their larger, $100 \mathrm{~km}$-sized, cousins. The upper and lower red curves correspond to our upper and lower $95 \%$ confidence level, which are derived without assuming any size distribution. This limit and the red point would be a factor of 2.4 lower if sub-kilometersized KBOs would have an ecliptic latitude distribution that is close to uniform for $-20^{\circ}<b<20^{\circ}$. The jump between $500 \mathrm{~m}$ and $600 \mathrm{~m}$ in the upper limit curve is due to the fact that below $500 \mathrm{~m}$ it is calculated for two events, whereas above $600 \mathrm{~m}$ for no events. The $95 \%$ upper limit from TAOS (Bianco et al. 2010) is about a factor of two lower than the one derived from our FGS survey, if sub-kilometer-sized KBOs have the same ecliptic latitude distribution as their larger, 100-km-sized, counterparts, and about the same if they follow a uniform ecliptic latitude distribution between $-20^{\circ}$ and $+20^{\circ}$.

Assuming that the $\mathrm{KBO}$ size distribution can be well described by a single power law that is parameterized by $N(>r) \propto$ $r^{1-q}$, where $N(>r)$ is the number of KBOs with radii greater than $r$, and $q$ is the power-law index, we can use the aboveestimated KBO abundances to calculate the power-law index below the observed break in the KBO size distribution. Assuming a break radius of $45 \mathrm{~km}$ and a corresponding cumulative

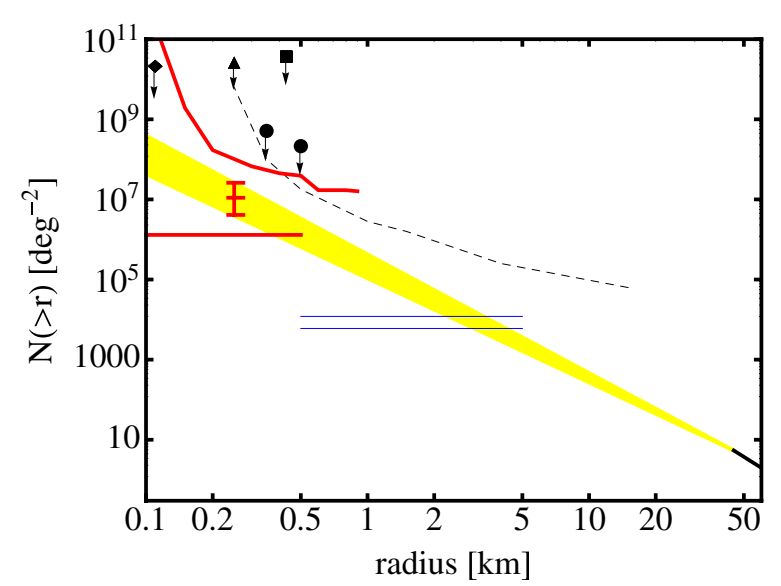

Figure 15. Cumulative KBO size distribution as a function of KBO radius. The results from our FGS survey are presented in three different ways: (i) the red point plotted at $r=250 \mathrm{~m}$ with the upper and lower error bars gives the best estimate of the KBO surface density around the ecliptic $\left(-5^{\circ}<b<5^{\circ}\right)$ from our survey with $1 \sigma$ errors, assuming that sub-kilometer-sized KBOs follow the same inclination distribution as their larger, 100-km-sized, cousins. (ii) The upper and lower red curves correspond to our upper and lower 95\% confidence level. (iii) The $1 \sigma$ range for our best estimate of the power-law size distribution index, $q=3.8 \pm 0.2$ is given by the yellow shaded region normalized to $N(>r)=5.4 \mathrm{deg}^{-2}$ at a radius of $45 \mathrm{~km}$ (Fuentes et al. 2009). The plotted red point, the upper and lower limits, and the power-law size distributions were all derived assuming that sub-kilometer-sized KBOs have the same ecliptic latitude distribution as their larger, 100-km-sized, counterparts. All would be a factor of 2.4 lower if sub-kilometer-sized KBOs would have an ecliptic latitude distribution that is close to uniform for $-20^{\circ}<b<20^{\circ}$. The area between the two blue horizontal lines defines the required scattered disk KBO abundances from Volk \& Malhotra (2008) in order to supply the Jupiter Family comets. In addition, 95\% upper limits from various optical KBO occultation surveys are shown as black symbols with arrows (circles: Bianco et al. 2009, square: Bickerton et al. 2008, triangle: Wang et al. 2010, and diamond: Roques et al. 2006) and as a dashed back line for TAOS (Bianco et al. 2010).

(A color version of this figure is available in the online journal.)

KBO surface density of $5.4 \mathrm{deg}^{-2}$ (Fuentes et al. 2009), we find $q=3.6 \pm 0.2$ and $q=3.8 \pm 0.2$ for a uniform KBO ecliptic latitude distribution and a KBO latitude distribution from Elliot et al. (2005), respectively.

Trilling et al. (2010) found that in contrast to larger KBOs with sizes above the break radius, fainter $(R>26)$ KBOs, which have sizes below the break radius, are dominated by dynamically excited objects $\left(i>5^{\circ}\right)$. If this result applies all the way to subkilometer-sized KBOs, then this suggests that the inclination distribution for sub-kilometer-sized KBOs may be dominated by dynamically excited objects and that the true abundance of subkilometer-sized KBOs, and their corresponding size distribution power-law index, may in fact lie somewhere between $N(r>$ $250 \mathrm{~m})=4.4 \times 10^{6}-1.1 \times 10^{7} \mathrm{deg}^{-2}$, and $q=3.6-3.8$, that we estimated above (see Figure 15). In addition, we can rule out a single power law below the break with $q>4.0$ at $2 \sigma$.

\subsection{Conclusion}

We presented here the analysis of $\sim 19,500$ new star hours of low ecliptic latitude $\left(|b| \leqslant 20^{\circ}\right)$ archival data that was obtained by the HST over a time span of more than nine years. Our search for stellar occultations by small KBOs yielded one new candidate event, which, assuming a circular orbit, corresponds to a body with a $\sim 500 \mathrm{~m}$ radius located at a distance of about 40 AU. Using bootstrap simulations, we estimate a probability of $\approx 5 \%$ that this event is due to random statistical fluctuations within the analyzed data set. Combining this new event with the 
single KBO occultation reported by Schlichting et al. (2009) we show that their ecliptic latitudes of 6.6 and 14.4 , respectively, are consistent with the observed inclination distribution of larger, 100-km-sized KBOs.

Assuming that the new candidate event and the event previously reported by Schlichting et al. (2009) are indeed genuine KBO occultations and that small, sub-kilometer-sized KBOs have the same ecliptic latitude distribution as larger KBOs, we find an ecliptic surface density of KBOs with radii larger than $250 \mathrm{~m}$ of $1.1_{-0.7}^{+1.5} \times 10^{7} \mathrm{deg}^{-2}$. If sub-kilometer-sized KBOs instead have a uniform ecliptic latitude distribution for $-20^{\circ}<b<20^{\circ}$ we find $N(r>250 \mathrm{~m})=4.4_{-2.8}^{+5.8} \times 10^{6} \mathrm{deg}^{-2}$. The ecliptic latitudes of the two events are consistent with both a uniform ecliptic latitude distribution and the ecliptic latitude distribution of larger KBOs published by Elliot et al. (2005). These estimated KBO abundances provide the best measurements of the abundance of sub-kilometer-sized KBOs to date and are, although consistent within $1 \sigma$, about a factor of two lower than previous results published by Schlichting et al. (2009).

Assuming that the $\mathrm{KBO}$ size distribution for bodies with radii smaller than the break radius can be well described by a single power law that is parameterized by $N(>r) \propto r^{1-q}$, where $N(>r)$ is the number of KBOs with radii greater than $r$, and $q$ is the power-law index, we find $q=3.6 \pm 0.2$ and $q=3.8 \pm 0.2$ for a uniform $\mathrm{KBO}$ ecliptic latitude distribution and a KBO ecliptic latitude distribution that follows the observed distribution for large KBOs, respectively. These results are consistent with a power-law index of $q=3.5$, corresponding to a collisional cascade consisting of material strength dominated bodies (Dohnanyi 1969) that all have the same constant velocity dispersion, within better than $1 \sigma$ and $2 \sigma$, respectively. We caution however that the actual size distribution of small KBOs is likely to exhibit significant deviations from a single power law due to possible changes from gravity to martial strength dominated bodies and waves that may exist in the small KBO size distribution (e.g., O'Brien \& Greenberg 2003; Pan \& Sari 2005). In addition, the velocity dispersion of the bodies in the cascade may not be constant and instead evolve as a function of size, which results in a size distributions that is significantly steeper than the one derived without velocity evolution (e.g., the standard $q=3.5$ power-law index of the Dohnanyi differential size spectrum can change to an index as large as $q=4$; Pan \& Schlichting 2012).

Finally, our findings suggest that small KBOs are numerous enough to satisfy the required supply rate for the Jupiter family comets calculated by Volk \& Malhotra (2008) for scattered disk objects. We can rule out a single power law below the break with $q>4.0$ at $2 \sigma$, confirming a strong deficit of sub-kilometer-sized KBOs compared to a population extrapolated from objects with $r>45 \mathrm{~km}$. This suggests that small KBOs are undergoing collisional erosion and that the Kuiper Belt is a true analog to the dust producing debris disks observed around other stars.

We thank Dr. Evan Kirby for analyzing and fitting the guide star spectrum. For H.S. support for this work was provided by NASA through Hubble Fellowship Grant No. HST-HF-51281.01-A awarded by the Space Telescope Science Institute, which is operated by the Association of Universities for Research in Astronomy, Inc., for NASA, under contact NAS 5-26555. R.S. acknowledges support by an ERC grant, a Packard Fellowship, and HST Grant No. HST-AR-12154.08-A. E.O.O. is incumbent of the Arye Dissentshik career development chair and is grateful for support by a grant from the Israeli Ministry of Science and for support from the The Helen Kimmel Center for Planetary Science.

\section{REFERENCES}

Alcock, C., Dave, R., Giammarco, J., et al. 2003, Earth Moon Planets, 92, 459

Axelrod, T. S., Alcock, C., Cook, K. H., \& Park, H.-S. 1992, in ASP Conf. Ser. 34, Robotic Telescopes in the 1990s, ed. A. V. Filippenko (San Francisco, CA: ASP), 171

Bailey, M. E. 1976, Nature, 259, 290

Benedict, G. F., McArthur, B. E., Feast, M. W., et al. 2007, AJ, 133, 1810 Bernstein, G. M., Trilling, D. E., Allen, R. L., et al. 2004, AJ, 128, 1364 Bianco, F. B., Protopapas, P., McLeod, B. A., et al. 2009, AJ, 138, 568 Bianco, F. B., Zhang, Z.-W., Lehner, M. J., et al. 2010, AJ, 139, 1499 Bickerton, S. J., Kavelaars, J. J., \& Welch, D. L. 2008, AJ, 135, 1039 Chang, H.-K., King, S.-K., Liang, J.-S., et al. 2006, Nature, 442, 660 Chang, H.-K., Liu, C.-Y., \& Chen, K.-T. 2011, MNRAS, 411, 427

Davis, D. R., \& Farinella, P. 1997, Icarus, 125, 50

Dohnanyi, J. W. 1969, J. Geophys. Res., 74, 2531

Doressoundiram, A., Liu, C.-Y., \& Roques, F. 2012, in Lunar and Planetary Inst. Technical Report, Vol. 43, Lunar and Planetary Institute Science Conference Abstracts, 1967

Dyson, F. J. 1992, QJRAS, 33, 45

Efron, B. 1982, in The Jackknife, the Bootstrap and Other Resampling Plans, ed. B. Efron (Philadelphia, PA: Society for Industrial Mathematics)

Elliot, J. L., Kern, S. D., Clancy, K. B., et al. 2005, AJ, 129, 1117

Fraser, W. C., Kavelaars, J. J., Holman, M. J., et al. 2008, Icarus, 195, 827

Fuentes, C. I., George, M. R., \& Holman, M. J. 2009, ApJ, 696, 91

Fuentes, C. I., \& Holman, M. J. 2008, AJ, 136, 83

Fuentes, C. I., Holman, M. J., Trilling, D. E., \& Protopapas, P. 2010, ApJ, 722,1290

Gilliland, R. L., McCullough, P. R., Nelan, E. P., et al. 2011, ApJ, 726, 2

Jewitt, D., Luu, J., \& Marsden, B. G. 1992, IAU Circ., 5611, 1

Jones, T. A., Levine, A. M., Morgan, E. H., \& Rappaport, S. 2008, ApJ, 677, 1241

Kenyon, S. J. 2002, PASP, 114, 265

Kenyon, S. J., \& Bromley, B. C. 2004, AJ, 128, 1916

Kenyon, S. J., \& Luu, J. X. 1999, AJ, 118, 1101

Nelan, E. P., Walborn, N. R., Wallace, D. J., et al. 2004, AJ, 128, 323

Noll, K. S., Grundy, W. M., Chiang, E. I., Margot, J.-L., \& Kern, S. D. 2008, in Binaries in the Kuiper Belt (The Solar System Beyond Neptune), ed. M. A. Barucci, H. Boehnhardt, D. P. Cruikshank, \& A. Morbidelli (Tucson, AZ: Univ. Arizona Press), 345

O’Brien, D. P., \& Greenberg, R. 2003, Icarus, 164, 334

Pan, M., \& Sari, R. 2005, Icarus, 173, 342

Pan, M., \& Schlichting, H. E. 2012, ApJ, 747, 113

Petit, J.-M., Kavelaars, J. J., Gladman, B. J., et al. 2011, AJ, 142, 131

Roques, F., Doressoundiram, A., Dhillon, V., et al. 2006, AJ, 132, 819

Schlichting, H. E., Ofek, E. O., Wenz, M., et al. 2009, Nature, 462, 895

Schlichting, H. E., \& Sari, R. 2011, ApJ, 728, 68

Sheinis, A. I., Bolte, M., Epps, H. W., et al. 2002, PASP, 114, 851

Skrutskie, M. F., Cutri, R. M., Stiening, R., et al. 2006, AJ, 131, 1163 Stern, S. A. 1996, AJ, 112, 1203

Trilling, D. E., Fuentes, C. I., \& Holman, M. J. 2010, ApJ, 724, L22

Volk, K., \& Malhotra, R. 2008, ApJ, 687, 714

Wang, J.-H., Protopapas, P., Chen, W.-P., et al. 2010, AJ, 139, 2003 